Spontaneous and persistent currents in superconductive and mesoscopic structures (Review)

I. O. Kulik

Citation: Low Temperature Physics 30, 528 (2004); doi: 10.1063/1.1789111

View online: http://dx.doi.org/10.1063/1.1789111

View Table of Contents: http://aip.scitation.org/toc/ltp/30/7

Published by the American Institute of Physics

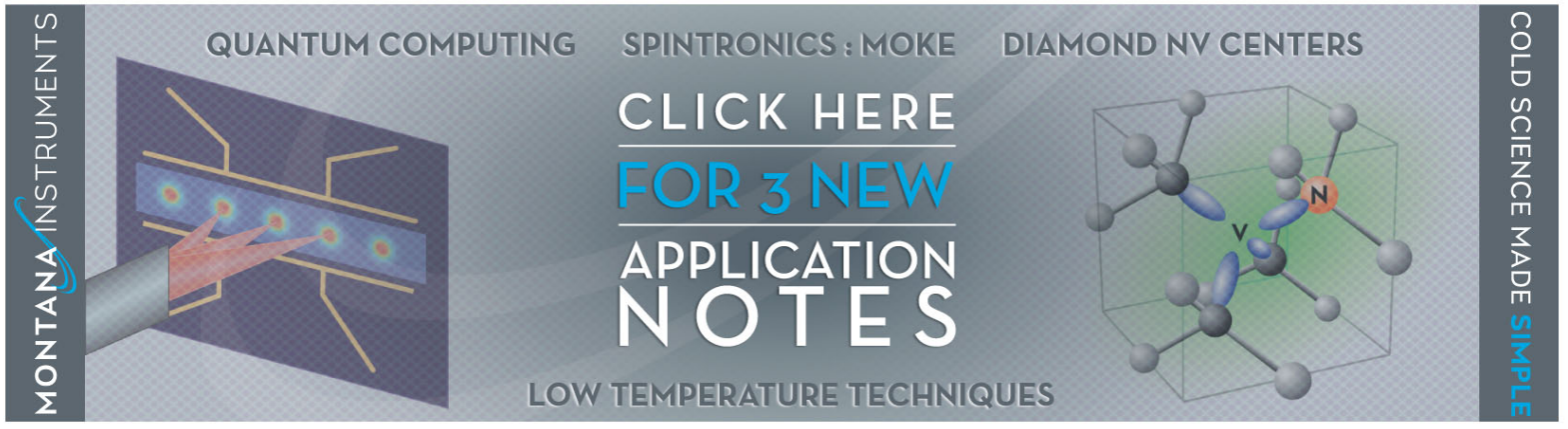




\title{
Spontaneous and persistent currents in superconductive and mesoscopic structures (Review)
}

\author{
I. O. Kulik*
}

Department of Physics, Bilkent University, Ankara 06533, Turkey; Physics Department, Stony Brook University, Stony Brook, New York 11794, USA; B. Verkin Institute for Low Temperature Physics and Engineering of the National Academy of Sciences of Ukraine, 47 Lenin Ave., Kharkov 61103, Ukraine (Received February 4, 2004)

Fiz. Nizk. Temp. 30, 705-713 (July-August 2004)

\begin{abstract}
We briefly review aspects of superconductive persistent currents in Josephson junctions of the $\mathrm{S} / \mathrm{I} / \mathrm{S}, \mathrm{S} / \mathrm{O} / \mathrm{S}$ and $\mathrm{S} / \mathrm{N} / \mathrm{S}$ types, focusing on the origin of jumps in the current versus phase dependences, and discuss in more detail the persistent and the "spontaneous" currents in Aharonov-Bohm mesoscopic and nanoscopic (macromolecular) structures. A fixednumber-of-electrons mesoscopic or macromolecular conducting ring is shown to be unstable against structural transformation removing spatial symmetry (in particular, azimuthal periodicity) of its electron-lattice Hamiltonian. In the case when the transformation is blocked by strong coupling to an external azimuthally symmetric environment, the system becomes bistable in its electronic configuration at a certain number of electrons. Under such a condition, the persistent current has a nonzero value even at an (almost) zero applied Aharonov-Bohm flux and results in very high magnetic susceptibility $d M / d H$ at small nonzero fields, followed by an oscillatory dependence at larger fields. We tentatively assume that previously observed oscillatory magnetization in cyclic metallo-organic molecules by Gatteschi et al. can be attributed to persistent currents. If this proves correct, it may present an opportunity for (and, more generally, macromolecular cyclic structures may suggest the possibility of) engineering quantum computational tools based on the Aharonov-Bohm effect in ballistic nanostructures and macromolecular cyclic aggregates. (C) 2004 American Institute of Physics.
\end{abstract}

[DOI: 10.1063/1.1789111]

\section{SUPERCONDUCTIVE WEAK LINKS}

Current can flow in a dissipationless manner under the control of an external parameter, the Josephson phase across a superconductive weak link ${ }^{1,2}$ (Fig. 1a) or a phase difference along a mesoscopic normal-metallic $\operatorname{loop}^{3-5}$ (Fig. 1b). In both cases, the phase is related to the magnetic flux piercing the loop. The flux can be considered as one created by a thin, infinitely long solenoid producing no magnetic field outside its interior (and therefore in a loop) but nevertheless affecting the quantum states of electrons in the loop. This nonlocal effect of magnetic flux on quantum states is known as the Aharonov-Bohm effect. ${ }^{6}$ The phase shift $\varphi$ due to magnetic flux $\Phi=\oint \mathbf{A} \cdot d \mathbf{l}$ is equal to

$$
\varphi=2 \pi \frac{\Phi}{\Phi_{0}}
$$

where $\Phi_{0}=h c / e^{*}$ is the flux quantum. In the Josephson junction, $\varphi$ is the phase of the pair wave function, and the effective charge $e^{*}$ equals twice the charge of the electron, $e^{*}=2 e$. In case of a normal-metal ring, $e^{*}$ is a singleelectron charge, $e$.

The current in a loop can be calculated as the derivative of the energy of the junction with respect to $\varphi$,

$$
J=\frac{e^{*}}{\hbar} \frac{\partial \mathrm{E}}{\partial \varphi}
$$

Superconductive junction theory considers contact types $\mathrm{S} / \mathrm{I} / \mathrm{S}$ (tunnel junctions), ${ }^{7}$ orifice-type contacts $\mathrm{S} / \mathrm{O} / \mathrm{S},{ }^{8}$ and the superconductor-normal metal-superconductor contacts S/N/S. ${ }^{9-11}$ The S/O/S and S/N/S contacts can include barriers at the interface between superconducting electrodes or inside the normal metal, respectively. The zero-temperature feature of the current-phase relation on which we will focus our attention is the existence of jumps at certain values of $\varphi$, in particular at $\varphi=\pi$ or $\varphi=0$. In the latter case (which is in effect a property of the Aharonov-Bohm weakly coupled loop considered in the next Section), the current assumes a nonzero value at zero flux. Jumps in $J(\varphi)$ in superconductive contacts are eliminated by the adjustment of the electronic system to the appropriate value of the gap parameter $\Delta(r)$. In the Aharonov-Bohm loop the adjustment will be achieved by the rearrangement of atoms in the loop (the Peierls or the Jahn-Teller effects, or more complex lattice transformation).

The Ambegaokar-Baratoff and Kulik-Omelyanchouk theories resulted in an interpolated current-phase relation suggested by Arnold $^{12}$ (see also the review ${ }^{13}$ )

$$
J(\varphi)=\frac{\pi \Delta_{0}}{2 e R_{0}} \frac{\sin \varphi}{\sqrt{r^{2}+t^{2} \cos ^{2}(\varphi / 2)}},
$$

where $R_{0}$ is the resistance of the junction in the normal state and $r^{2}$ and $t^{2}$ are the reflection and transmission probabilities (with $r^{2}+t^{2}=1$ ) in the normal state. $\Delta_{0}$ is the order param- 

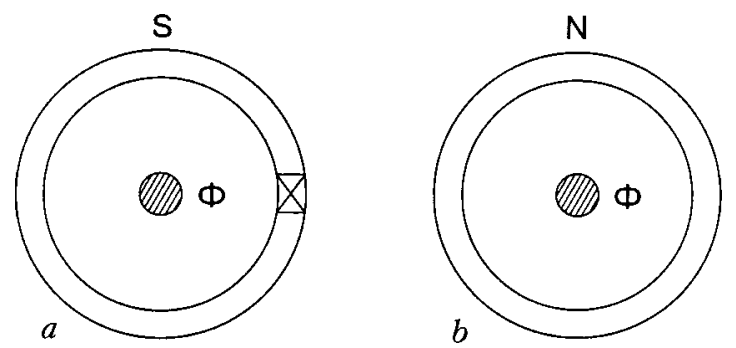

FIG. 1. Superconducting loop with a weak contact (crossed) (a). Normalmetal mesoscopic loop carrying current $J(b)$.

eter of the superconductor (the BCS energy gap at $T=0$ ). At $t \ll 1$, formula (3) reduces to the Ambegaokar-Baratoff relation

$$
J_{A B}=\frac{\pi \Delta_{0}}{2 e R_{0}} \sin \varphi
$$

whereas at $r=0$ (no barrier) it gives the KulikOmelyanchouk formula

$$
J_{K O}=\frac{\pi \Delta_{0}}{e R_{0}} \sin (\varphi / 2), \quad-\pi<\varphi<\pi
$$

corresponding to twice as large a critical current at the same value of the contact resistence. The energy versus phase relation in the $\mathrm{S} / \mathrm{O} / \mathrm{S}$ structure with barriers is given by

$$
E_{S O S}=-\frac{\pi \hbar \Delta_{0}}{2 e^{2} R_{0} t^{2}} \sqrt{r^{2}+t^{2} \cos ^{2}(\varphi / 2)}
$$

and is presented in Fig. 2. The S/N/S junction is represented by the $E(\varphi)$ dependence at $T=0$

$$
E_{S N S}=-\frac{\hbar v_{F}}{6 d} N_{\perp} t^{2}\left[1-\left(\frac{\varphi \bmod 2 \pi}{\pi}\right)^{2}\right]
$$

where $v_{F}$ is the Fermi velocity of the metal and $N_{\perp}$ $=S k_{F}^{2} / 4 \pi$ is the number of perpendicular conducting channels in the normal bridge between superconductors of length $d$ and cross section $S$. From the above expression, the current in the $\mathrm{S} / \mathrm{N} / \mathrm{S}$ structure at $T=0$ becomes

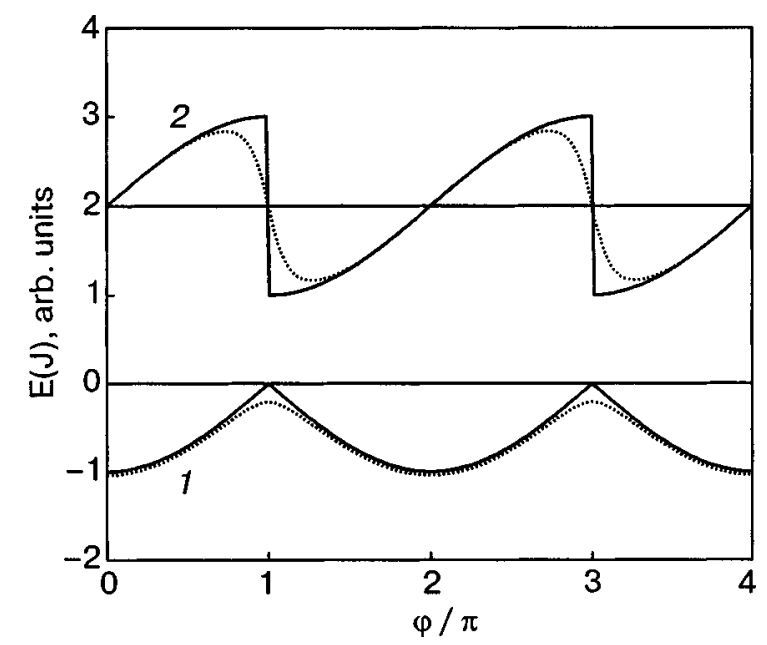

FIG. 2. Energy of the $\mathrm{S} / \mathrm{O} / \mathrm{S}$ contact versus phase at $T=0$ (1). Supercurrent versus phase (2). The solid curves correspond to $r=0$, the dotted curves to $r=0.2$. The $J(\varphi)$ curves are shifted upward arbitrarily for clarity.

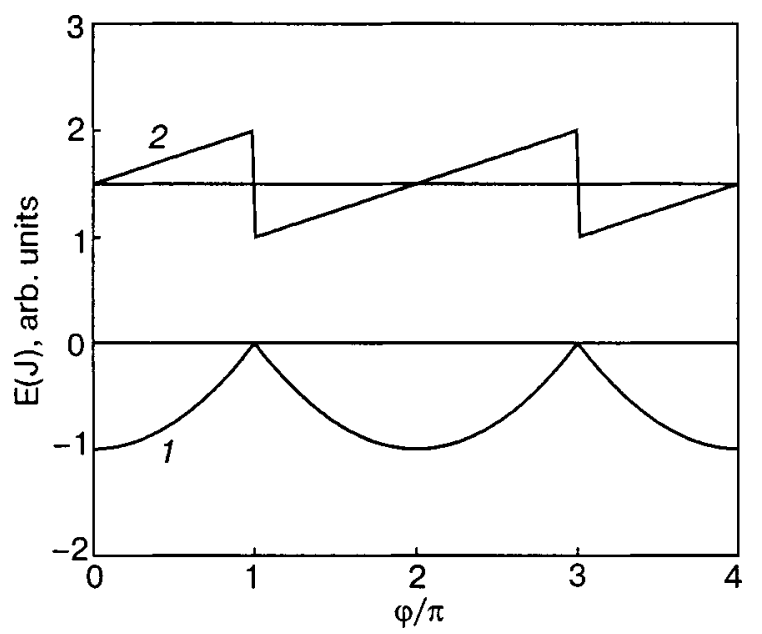

FIG. 3. Energy of the S/N/S contact versus phase at $T=0(1)$. Supercurrent versus phase (2). The $J(\varphi)$ curve is shifted upward arbitrarily for clarity.

$$
J(\varphi) \approx \frac{2 \hbar v_{F}}{3 \pi e R_{0} d} \varphi, \quad-\pi<\varphi<\pi
$$

and is presented in Fig. 3 together with the $E(\varphi)$ dependence.

\section{PERSISTENT CURRENTS IN MESOSCOPIC SYSTEMS}

Persistent currents (first discovered and termed nondecaying currents ${ }^{4}$ ) have been predicted for mesoscopic conducting loops ${ }^{3-5}$ which do not show the effect of superconductivity. The current appears in the presence of magnetic field as a result of the Aharonov-Bohm effect. ${ }^{6}$ As discussed in a review paper, ${ }^{14}$ persistent currents are similar to the orbital currents in normal metals first considered by Teller ${ }^{15}$ in his interpretation of Landau diamagnetism in metals, ${ }^{16}$ but specific to the doubly connected geometry of the conductors (loops, hollow cylinders, etc.). Observations of persistent currents have been made in indirect ${ }^{17,18}$ as well as in direct $^{19-21}$ experiments, showing single-flux-quantum $\Phi_{0}$ $=h c / e$ periodicity in the resistance of thin $\mathrm{Nb}$ wires ${ }^{17}$ and networks of isolated $\mathrm{Cu}$ rings, ${ }^{18}$ and in single-loop experiments on metals, ${ }^{19}$ semiconductors, ${ }^{20}$ and macromolecular metallo-organic compounds. ${ }^{21}$ Contrary to the authors of Ref. 21 (an interpretation of magnetic oscillation ${ }^{21}$ based on antiferromagnetic ordering of $\mathrm{Fe}$ ions in a "ferric wheel" $\left.\left[\mathrm{Fe}(\mathrm{OMe})_{2}\left(\mathrm{O}_{2} \mathrm{CCH}_{2} \mathrm{Cl}\right)\right]_{10}\right)$, we propose that the $6 T$ periodic magnetization in this compound is due to Aharonov-Bohm persistent current flowing in the outer ring of $\mathrm{O}$ atoms while the inner ring of $\mathrm{Fe}$ atoms serves as a concentrator of magnetic field to the center of the ring. In Ref. 22 the $8 T$-periodic variation of resistivity in molecular conducting cylinders (carbon nanotubes) was attributed to the Altshuler-Aronov-Spivak effect, ${ }^{23}$ a companion effect to the classical Aharonov-Bohm mechanism but with a twice smaller periodicity in magnetic flux $\Delta \Phi=h c / 2 e$.

Aspects of the Aharonov-Bohm persistent currents in complex and correlated systems have been considered in various papers, in particular by studying the strong coupling $^{24-26}$ and localization ${ }^{27,28}$ effects, thermodynamicstatistical properties, ${ }^{29-31}$ polaron effects, ${ }^{32,33}$ effects of strong magnetic field ${ }^{34,35}$ and spin-orbit interaction, ${ }^{36,37}$ 
Peierls transition, ${ }^{38-40}$ Wigner crystallization ${ }^{41}$ and Coulomb blockade, ${ }^{42}$ persistent current oscillation in hollow cylinders with toroidal geometry, ${ }^{43}$ nonequilibrium and timedependent effects, ${ }^{44-48}$ weak links in the loop, ${ }^{49}$ as well as the nontraditional phase effects (geometrical and Berry's phase, instantons, etc. $)^{50-53}$ summarized in recent reviews. ${ }^{14,54-57}$ Further trends in the macromolecular persistent and spontaneous currents ${ }^{58-60}$ include quantum computational ${ }^{61}$ prospects of using Aharonov-Bohm loops as quantum bits (qubits) with the advantages of easier (radiation-free) manipulation of qubit states and increased decoherence times as compared to macroscopic "Schrödinger cat" structures (Josephson junctions). The smallest (three-site) persistent current ring displays a $\Lambda$-shaped energy configuration ${ }^{59}$ with two degenerate ground states at external flux $\Phi_{0}=h c / 2 e$. The spontaneous persistent current loop will achieve the degenerate state at zero field or, if the degeneracy is lifted by the electron-phonon coupling, at a reasonably low field.

Persistent current is a voltage-free nondecaying current which exists as a manifestation of the fact that the ground state of a doubly connected conductor in a magnetic field is a current-carrying one. This statement has been proved for ballistic loops ${ }^{4}$ and for diffusive rings. ${ }^{5}$ There is no fundamental difference between these two extremes. Counterintuitively, ballistic structure does not show infinite conductivity, as has sometimes been naively supposed; the dc resistance of the loop is infinite rather than zero when a dc electric field is applied to the system. In the case when a current is fed through the structure, no voltage appears provided that the magnitude of the current is smaller than a certain critical value. This applies to both elastic and inelastic scattering. The magnitude of the critical current of the ballistic ring smoothly matches the current of the diffusive ring when the mean free path $l$ becomes large. In the dirty limit, $l \ll L$, where $L$ is the ring circumference, the critical value of the supercurrent decreases proportionally to $l / L$ according to Ref. 62, or to $(l / L)^{1 / 2}$ according to a numerical simulation. ${ }^{14}$ The nondecaying current does not even require severe restriction on the so-called "phase breaking" electron mean free path. In fact, the normal-metal supercurrent is an analog of the "incoherent" Josephson effect, ${ }^{63,64}$ in which the phase of the superconductor is considered as a classical variable. Stronger criteria (that the dephasing length is larger than the system size, and the analogous requirement in the time domain, that the "decoherence time" is larger than the characteristic time of observation) apply to persistent current rings as quantum computational tools mentioned above, which are the analogs of the macroscopic quantum tunneling. ${ }^{65-67}$

\section{SPONTANEOUS PERSISTENT CURRENTS}

Persistent current appears in a ballistic ring due to the Aharonov-Bohm field. The current, however, can also originate when the external field is zero- the "spontaneous" current. This situation has been noticed accidentally by various authors, in particular, in Refs. 68-70, but it has not seemed convincing due to the fixed-chemical-potential configuration, and it has been attributed to the effect of Peierls instability in the ring ${ }^{40}$ (criticized in Refs. 71 and 72 in regard to the inaccuracy of the mean field approximation). In fact, the

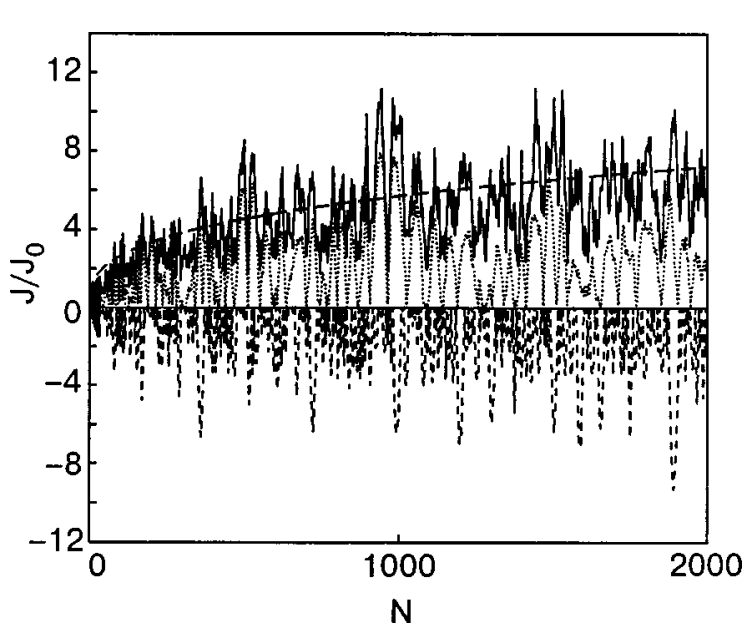

FIG. 4. Persistent current versus number of electrons in a ring with a ratio of cross-sectional dimensions $L: L_{1}: L_{2}=10: 1: 1$ (spinfull configuration). The upper curve is the maximum current in units of $J_{0}=e v_{F} / L$ at given $N$, the dotted curve is the amplitude of the first harmonic of $J_{\text {pers }}(\Phi)$, and the curve at negative $J$ is the spontaneous persistent current as defined below, also in units of $J_{0}$. The dashed curve is the square root of the number of perpendicular channels $N_{\perp}$ plotted against $N$.

fixed-number-of-particles ring with an odd number of electrons displays a number of structural instabilities: the Peierls transformation $^{73}$ and the Jahn-Teller effect ${ }^{74}$ are the bestknown examples, and the (generally more complex) atomic rearrangement when the ground state proves degenerate in a symmetric configuration.

In Fig. 4 we show the dependence of the maximal persistent current, as well as the spontaneous current, on the number of electrons in a ring which was modeled as a finitelength hollow cylinder with rectangular cross section $L_{1}$ $\times L_{2}$ containing a finite number of perpendicular electron channels $N_{\perp}=L_{1} L_{2} k_{F}^{2} / 2 \pi^{2}$. Note that the magnitude of the current in a ballistic ring is not $e v_{F} / L$, as is sometimes suggested $\left(v_{F}\right.$ is the Fermi velocity), but rather approaches a value $J_{\max } \sim\left(e v_{f} / L\right) N_{\perp}^{1 / 2}$ (see Ref. 4). The dependence $J_{\max }(N)$ at $T=0$ is irregular due to the addition of negative and positive currents from different electron eigenstates in longitudinal and transverse channels.

Figure 5 shows the bistability effect in a ring. While at

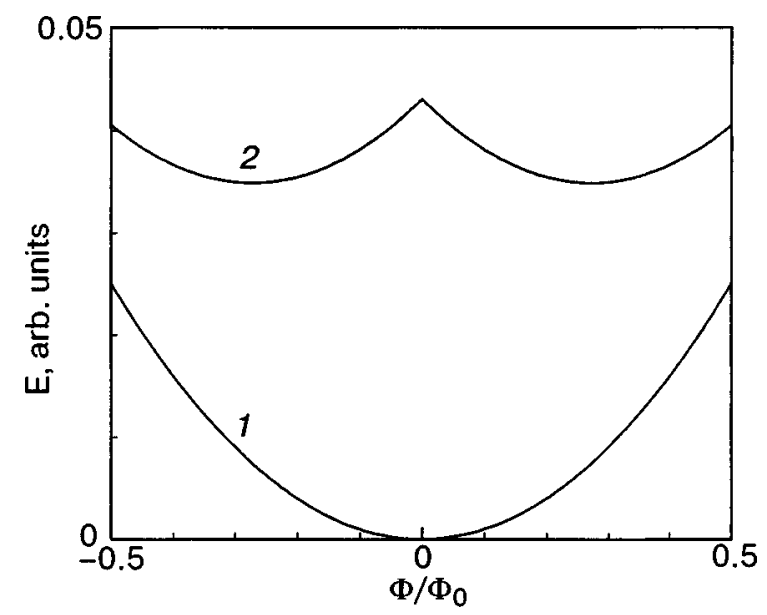

FIG. 5. Bistable configuration in a ring: Energy versus flux in a ring of 10 electrons (1) and 11 electrons (2). The second curve is shifted downward for convenience (but not rescaled). 
an even number of electrons the electronic energy has a minimum at $\Phi=0$, it acquires a maximum when the number of electrons is odd. (The inductive energy, to be included below, will shift the position of the minima in curve 2 of Fig. 5 to the origin, so that a degenerate state will appear in the near vicinity of $\Phi=0$.)

The spontaneous current has the same order of magnitude as the maximal persistent current and represents an inseparable part of the Aharonov-Bohm effect. The structural transformation is investigated below in an exact way by considering the ring dynamics in the tight binding approximation. The "lattice" (the atomic configuration of the loop) can respond to the degenerate ground state by making an atomic readjustment similar to the Peierls transition (doubling of the lattice period in a one-dimensional atomic chain; see, e.g., Refs. 75 and 76), or a more complex atomic rearrangement.

In fact, such a possibility clearly shows up in the case of a 1D loop with the discrete quantum states $(\theta$ is the azimuthal angle)

$$
\psi_{n}=\frac{1}{\sqrt{L}} \exp (\text { in } \theta)
$$

corresponding to energies

$$
\varepsilon_{n}=\frac{\hbar^{2}}{2 m R^{2}}(n-f)^{2},
$$

where $n=0, \pm 1, \pm 2, \ldots$ and $f=\Phi / \Phi_{0}$ is the magnetic flux threading the loop in units of the flux quantum $\Phi_{0}=4$ $\times 10^{-7} \mathrm{G} \cdot \mathrm{cm}^{2}$.

As an example, the loop with 3 electrons has energy

$$
E(f)=\varepsilon_{0}\left[f^{2}+\frac{1}{2}( \pm 1-f)^{2}\right]+\frac{\mathcal{L} J_{0}^{2}}{2 c^{2}} J^{2}(f)
$$

corresponding to two spin-1/2 states with $n=0$ and one state with $n=1$ or $n=-1$. The last term in Eq. (11) is the magnetic inductive energy and $\mathcal{L}$ is the inductance (of the order of the ring circumference, in the units adopted). The current $J=-(e / h) \partial E / \partial f$ is equal to

$$
J(f)=J_{0}( \pm 1-3|f|), \quad J_{0}=e \varepsilon_{0} / h
$$

and is nonzero at $f=0$ in either of the states \pm . The ratio of magnetic energy to kinetic energy is of order

$$
\eta=\frac{\mathcal{L} J_{0}^{2}}{2 c^{2} \varepsilon_{0}} \simeq \frac{e^{2}}{4 \pi m c^{2} R} \sim 10^{-6} \frac{a_{0}}{R},
$$

where $a_{0}$ is the Bohr radius. This is a very small quantity, and therefore the magnetic energy is unimportant in the energy balance of the loop. The flux in the loop equals $f$ $=f_{\text {ext }}+2 \eta j_{f}$, where $f_{\text {ext }}$ is an external flux and $j_{f}$ $=J(f) / J_{0}$. The correction for the externally applied flux is essential only at $f_{\text {ext }} \sim \eta$; otherwise, we can ignore this contribution.

The property of nonzero persistent current thus demonstrated for the noninteracting electrons survives strong electron-electron coupling but collapses when the coupling to the lattice is included (see below). Nevertheless, when the loop is on a rigid background (say, a cyclic molecule on a substrate of a much more rigidly bound solid) the degeneracy may not be lifted, or may remain in a very narrow interval of externally applied fields. We will investigate this possibility in the tight binding approximation, ${ }^{77,78}$ in which electrons are bound to certain atomic locations (traps) and make the loop conducting by resonant tunneling between these locations.

In the tight binding approximation, Hamiltonian of the loop in the second-quantized form reads

$$
\begin{aligned}
H= & \sum_{j=1}^{N}\left(t_{j} a_{j \sigma}^{+} a_{j+1, \sigma} e^{\left.i \alpha_{j}+\text { h.c. }\right)}+U \sum_{i=1}^{N} n_{i \uparrow} n_{i \downarrow}\right. \\
& +V \sum_{i=1, \sigma, \sigma^{\prime}}^{N} n_{i \sigma} n_{i+1, \sigma^{\prime}}+\frac{1}{2} K \sum_{j=1}^{N}\left(\theta_{j}-\theta_{j+1}\right)^{2},
\end{aligned}
$$

where $t_{j}$ is the hopping amplitude between two near configurational sites, $j$ and $j+1$,

$$
t_{j}=t_{0}+g\left(\theta_{j}-\theta_{j+1}\right), \quad n_{i \sigma}=a_{i \sigma}^{+} a_{i \sigma},
$$

and $\alpha_{j}$ is the Aharonov-Bohm phase (a Peierls substitution for the phase of hopping amplitude)

$$
\alpha_{j}=\frac{2 \pi f}{N}+\left(\theta_{j}-\theta_{j+1}\right) f .
$$

$a_{j \sigma}^{+}$is the creation (and $a_{j \sigma}$, the annihilation) operator of an electron at site $j$ with spin $\sigma, \theta_{j}, j=1,2, \ldots, N$ are the angles of distortion of site locations from their equilibrium positions $\theta_{j}^{0}=2 \pi j / N$ and satisfy the requirement $\sum_{j=1}^{N} \theta_{j}=0$, and $g$ is the electron-phonon coupling constant. The interaction (15) reflects the fact that the hopping amplitude depends on the distance between the localization positions and assumes that the displacement $\theta_{j}-\theta_{j+1}$ is small in comparison to $2 \pi / N$. $U$ and $V$ are Hubbard parameters of the on-site and intrasite interactions. The parameters are assumed such that system is not superconductive (e.g., $U>0$; and anyway, superconductivity is not allowed for a 1D system and is ruled out for a small system). The last term in Hamiltonian (14) is the elastic energy, and $K$ is the stiffness parameter of the lattice.

In the smallest loop, the one with three sites $(N=3)$, the only two free parameters of the lattice displacement, $X_{1}$ and $X_{2}$, are

$$
\theta_{1}=X_{1}+X_{2}, \quad \theta_{2}=-X_{1}+X_{2}, \quad \theta_{3}=-2 X_{2}
$$

which are decomposed to second-quantized Bose operators $b_{1}$ and $b_{2}$ according to

$$
X_{1}=\left(\frac{3 K}{\omega}\right)^{1 / 4}\left(b_{1}+b_{1}^{+}\right), \quad X_{2}=3\left(\frac{K}{3 \omega}\right)^{1 / 4}\left(b_{2}+b_{2}^{+}\right) \text {. }
$$

The system (14) is solved numerically with the ABC compiler, ${ }^{79}$ which includes the creation-annihilation operators as its parameter types. These are generated as compiler macros with sparse matrices

$$
\begin{aligned}
& A_{n}=C_{n}^{\left(N_{1}\right)} \otimes 1^{\left(N_{2}\right)} \text { fermionic sector } \\
& B_{n}=1^{\left(N_{1}\right)} \otimes C_{n}^{\left(N_{2}\right)} \text { bosonic sector, }
\end{aligned}
$$

where $1^{(N)}$ is a unit matrix of dimension $2^{N}$ and $C_{n}^{(N)}, n$ $=1, \ldots, N$ are Fermi/Bose operators in a space of the same dimension,

$$
C_{n}^{(N)}=(u \otimes)^{N-n} a(\otimes v)^{n-1},
$$




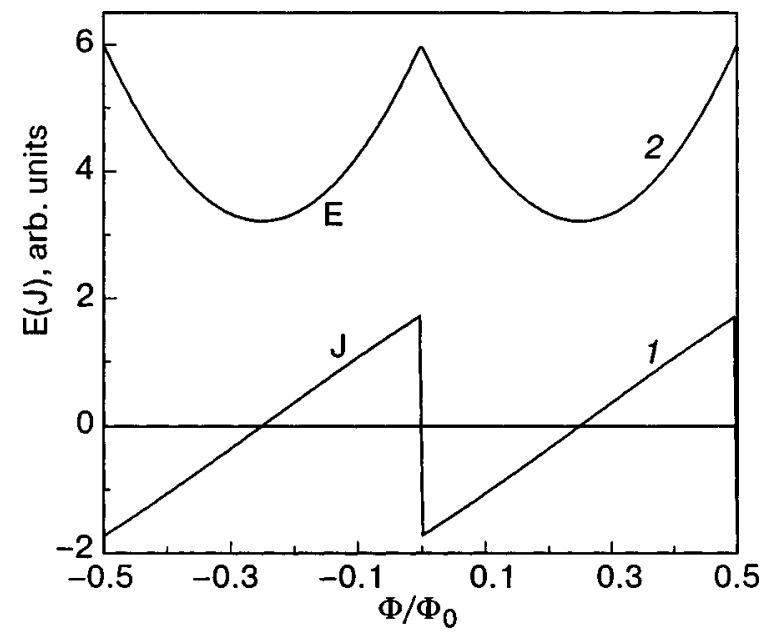

FIG. 6. Current versus magnetic flux in the 3-site loop with 3 noninteracting electrons (1). Energy versus flux for the $N=3, n=3$ loop at the value of the hopping parameter $t_{0}=-1(2)$. The energy is rescaled and arbitrarily shifted upward for clarity.

$a, u$, and $v$ are the $2 \times 2$ matrices $(\otimes$ is the symbol of the Kronecker matrix product)

$$
a=\left(\begin{array}{ll}
0 & 0 \\
1 & 0
\end{array}\right), \quad u=\left(\begin{array}{ll}
1 & 0 \\
0 & 1
\end{array}\right), \quad v=\left(\begin{array}{ll}
1 & 0 \\
0 & \eta
\end{array}\right),
$$

and $\eta$ is a parameter

$$
\eta=\left\{\begin{array}{cc}
-1 & \text { fermionic sector } \\
1 & \text { bosonic sector. }
\end{array}\right.
$$

Bosons are considered as "hard-core bosons," such that there are only two discrete states for each mode of displacement. We calculate the ground state of Hamiltonian (14) as a function of magnetic flux $f$ (a classical variable). In application to real atomic (macromolecular) systems, we can consider $X_{1}$ and $X_{2}$ as classical variables, since the quantum uncertainties in the coordinates $\left(\Delta X_{1,2} \sim(\hbar / M \omega)^{1 / 2}\right)$ are typically much smaller than the interatomic distances $(M$ is the mass of an atom and $\omega \sim 10^{13} \mathrm{~s}^{-1}$ is the characteristic vibration frequency). The energy of the loop is calculated as function of $X_{1}, X_{2}$ and further is minimized with respect to $X_{1}, X_{2}$ for each value of $f$. The nonzero values of $X_{1}, X_{2}$ will signify the "lattice" (the ionic core of the macromolecule) instability against the structural transformation which is analogous to the Peierls transition.

For the 3-site loop, the $E(f)$ dependence is shown in Fig. 6 together with the dependence of the current on $f$. The latter shows a discontinuity at $f=0$ of the same order of magnitude as the standard value of the persistent current. The current at $f=0$ is paramagnetic, since the energy versus flux has a maximum rather than a minimum at $f=0$. On-site interaction reduces the amplitude of the persistent current near zero flux (Fig. 7) but doesn't remove its discontinuity at $f$ $=0$. Therefore, the strongest opponent of the AharonovBohm effect, the electron-electron interaction, leaves it qualitatively unchanged.

On the other hand, the electron-phonon interaction flattens the $E(f)$ dependence near the peak value; see Fig. 8. At large stiffnesses $K$ this flattening remains important only for small magnetic fluxes, much smaller than the flux quantiza-

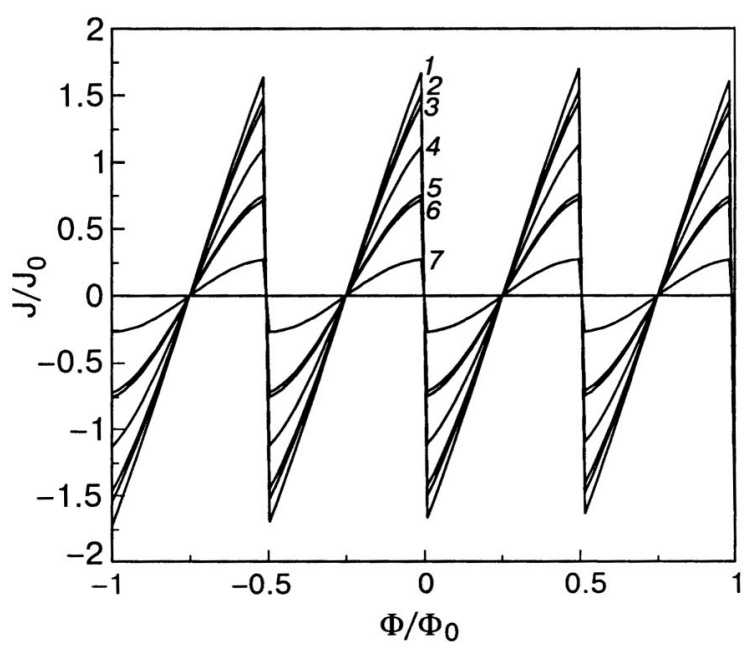

FIG. 7. Spontaneous persistent current versus flux for $t_{0}=-1$ and various values of the Hubbard parameter $U: 0(1) ;-2(2) ; 2(3) ;-5(4) ; 5(5) ;-10$ (6); 10 (7).

tion period $\Delta \Phi=\Phi_{0}$. Note that the persistent current peak is reduced in amplitude only slightly near $\Phi=0$. As is seen from Fig. 9, the electron-phonon interaction splits the singularity at $\Phi=0$ into two singularities at $\Phi= \pm \Phi_{\text {sing }}$. Outside the interval $-\Phi_{\text {sing }}<\Phi<\Phi_{\text {sing }}$ the structural transformation is blocked by the Aharonov-Bohm flux. The range of magnetic fluxes between $-\Phi_{\text {sing }}$ and $\Phi_{\text {sing }}$ determines the domain of the developing lattice transformation, which manifests itself in nonzero values of the lattice deformations $X_{1}, X_{2}$. The latter property allows us to suggest that the spontaneous persistent current state (a peak of dissipationless charge transport at, or near, zero flux) remains for nonzero flux when the electron-phonon coupling is not too strong or when the lattice stiffness is larger than certain critical value.

\section{CONCLUSION}

We have considered the Aharonov-Bohm effect in an angular-periodic macromolecular loop like, e.g., an aromatic cyclic molecule, and found that the Aharonov-Bohm flux applied to the loop arrests the lattice instability (rearrange-

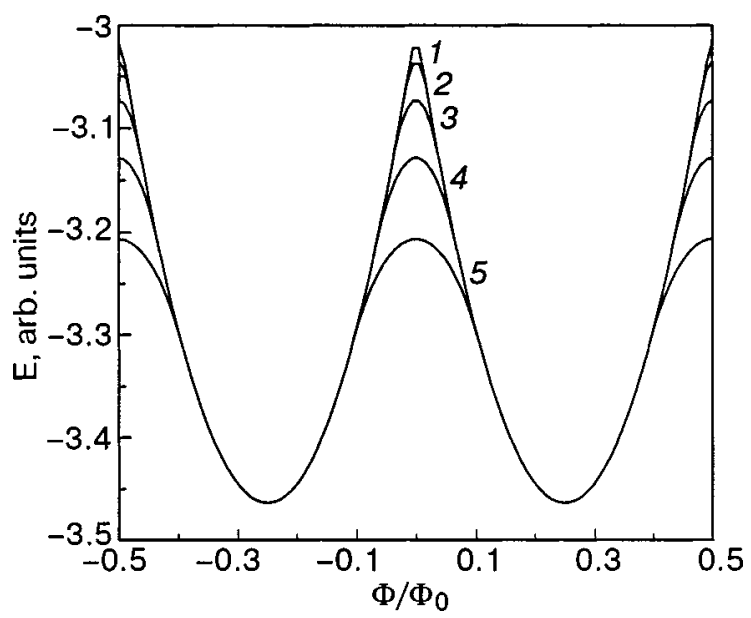

FIG. 8. Energy versus flux in a loop of noninteracting electrons coupled to the lattice with the value of the coupling parameter $g=1$ and various values of the stiffness parameter $K: 2(1) ; 3$ (2); 5 (3); 10 (4); 20 (5). 


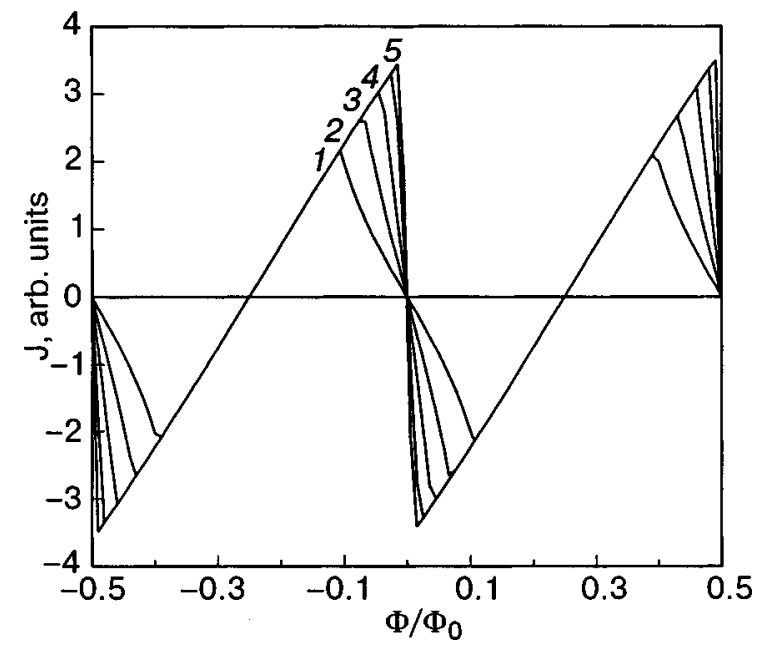

FIG. 9. Energy versus flux for a loop with coupling constant $g=1$ and various values of the stiffness $K: 2$ (1); 3 (2); 5 (3); 10 (4); 20 (5).

ment of molecular atoms or blocks within the molecule). This is a consequence of the fact that the weak-coupling effect of electron hopping between sites of electron localization cannot provide enough energy for initiating a shift of the atoms from periodic locations except at quite small magnetic fields. As a result, the ground state of the system at a certain electron concentration becomes current-carrying at zero (or very small) magnetic flux - a state with "spontaneous" persistent current. This effect suggests the possibility of using appropriately engineered macromolecular structures as elementary qubits, the degenerate or near-degenerate states sought for processing of quantum information. ${ }^{61}$ As was shown in Ref. 59, the three-site Aharonov-Bohm loop supports all logical operations (the quantum logic gates) required for quantum computation and quantum communication, which are effected by static voltages applied to the loop perpendicular to the magnetic flux and such that the loop is driven to a $\Lambda$-shaped energy configuration with the two degenerate ground states making elements of the qubit and the third, higher-energy state implementing radiation-free quantum logic gates. Very strong magnetic fields are required for the formation of such states (corresponding to a flux equal to half of the flux quantum). The spontaneous persistent currents discussed in the present paper allow one to reduce these fields by orders of magnitude.

*E-mail: iokulik@yahoo.com

${ }^{1}$ B. D. Josephson, Phys. Lett. 1, 251 (1962).

${ }^{2}$ B. D. Josephson, in Superconductivity, Vol. 1, edited by R. D. Parks, Marcell Dekker, New York (1969), p. 423.

${ }^{3}$ F. Bloch, Phys. Rev. B 2, 109 (1970) and references therein. This paper proved exact periodicity of the energy of a loop as a function of magnetic flux with a period hcle but with an indefinite amplitude.

${ }^{4}$ I. O. Kulik, JETP Lett. 11, 275 (1970).

${ }^{5}$ M. Buttiker, Y. Imry, and R. Landauer, Phys. Lett. A 96, 365 (1983).

${ }^{6}$ Y. Aharonov and D. Bohm, Phys. Rev. B 115, 485 (1959).

${ }^{7}$ V. Ambegaokar and A. Baratoff, Phys. Rev. Lett. 10, 486 (1963); 11, 104 (1963).

${ }^{8}$ O. Kulik and A. N. Omelyanchouk, Fiz. Nizk. Temp. 3, 945 (1977) [Sov. J. Low Temp. Phys. 3, 459 (1977)].

${ }^{9}$ O. Kulik, Zh. Éksp. Teor. Fiz. 57, 1745 (1969) [Sov. Phys. JETP 30, 944 (1970)]
${ }^{10}$ C. Ishii, Prog. Theor. Phys. 44, 1525 (1970); J. Bardeen and J. L. Johnson, Phys. Rev. B 5, 72 (1972); A. V. Swidzinsky, T. N. Antsygina, and E. N. Bratus, J. Low Temp. Phys. 10, 131 (1973).

${ }^{11}$ O. Cakir and I. O. Kulik, Phys. Rev. B 67, 174514 (2003).

${ }^{12}$ G. B. Arnold, J. Low Temp. Phys. 59, 143 (1985).

${ }^{13}$ S. Kasiwaya and Y. Tanaka, Prog. Theor. Phys. 63, 1641 (2000); A. Furusaki, H. Takayanagi, and M. Tsukada, Phys. Rev. B 45, 10563 (1992).

${ }^{14} \mathrm{O}$. Kulik, in Quantum Mesoscopic Phenomena and Mesoscopic Devices in Microelectronics, edited by I. O. Kulik and R. Ellialtioglu, Kluwer, Dordrecht (2000), p. 259.

${ }^{15}$ E. Teller, Z. Phys. 67, 311 (1931).

${ }^{16}$ L. D. Landau, Z. Phys. 64, 629 (1930).

${ }^{17}$ N. B. Brandt, E. N. Bogachek, D. V. Gitsu, G. A. Gogadze, I. O. Kulik, A. A. Nikolaeva, and Ya. G. Ponomarev, Fiz. Nizk. Temp. 8, 718 (1982) [Sov. J. Low Temp. Phys. 8, 358 (1982)]

${ }^{18}$ L. P. Levy, G. Dolan, J. Dunsmuir, and H. Bouchiat, Phys. Rev. Lett. 64, 2074 (1990).

${ }^{19}$ V. Chandrasekhar, R. A. Webb, M. J. Brady, M. B. Ketchen, W. J. Gallagher, and A. Kleinsasser, Phys. Rev. Lett. 67, 3578 (1991).

${ }^{20}$ D. Mally, C. Chapelier, and A. Benoit, Phys. Rev. Lett. 70, 2020 (1993).

${ }^{21}$ D. Gatteschi, A. Caneschi, L. Pardi, and R. Sessoli, Science 265, 1054 (1994); K. L. Taft, C. D. Delfs, G. C. Papaefthymiou, S. Foner, D. Gatteschi, and S. J. Lippard, J. Am. Chem. Soc. 116, 823 (1994).

${ }^{22}$ C. Schönenberger, A. Bachtold, and C. Strunk, J.-P. Salvetat, and L. Forro, Arrl. Phys. A 69, 283 (1999).

${ }^{23}$ B. L. Altshuler, A. G. Aronov, and B. Z. Spivak, [JETP Lett. 33, 94 (1981)].

${ }^{24}$ A. Ferretti, I. O. Kulik, and A. Lami, Phys. Rev. B 47, 12235 (1993).

${ }^{25}$ F. V. Kusmartsev, J. Phys.: Condens. Matter 3, 3199 (1991).

${ }^{26}$ M. V. Moskalets, Physica E 4, 111 (1999).

${ }^{27}$ I. O. Kulik, Fiz. Nizk. Temp. 13, 206 (1987) [Sov. J. Low Temp. Phys. 13, 115 (1987)].

${ }^{28}$ G. Kirczenow, J. Phys.: Condens. Matter 7, 2021 (1995).

${ }^{29}$ B. L. Altshuler, Y. Gefen, and Y. Imry, Phys. Rev. Lett. 66, 88 (1991).

${ }^{30}$ G. Montambaux, H. Bouchiat, D. Sigeti, and F. Friesner, Phys. Rev. B 42, 7647 (1990).

${ }^{31}$ J. F. Weisz, R. Kishore, and F. V. Kusmartsev, Phys. Rev. B 49, 8126 (1994).

${ }^{32}$ Yi-Chang Zhou, Xin-E Yang, and Huo-Zhong Li, Phys. Lett. A 190, 123 (1994).

${ }^{33}$ M. Bayindir and I. O. Kulik, in Quantum Mesoscopic Phenomena and Mesoscopic Devices in Microelectronics, edited by I. O. Kulik and R. Ellialtioglu, Kluwer, Dordrecht (2000), p. 283.

${ }^{34}$ D. Eliyahu, R. Berkovits, M. Abraham, and Y. Avishai, Phys. Rev. B 49, 14448 (1994).

${ }^{35}$ I. O. Kulik, Physica B 284 (2000).

${ }^{36}$ Y. Lyanda-Geller, Phys. Rev. Lett. 80, 4273 (1998).

${ }^{37}$ M. V. Moskalets, JETP Lett. 70, 602 (1999).

${ }^{38}$ I. O. Kulik, A. S. Rozhavsky, and E. N. Bogachek, JETP Lett. 47, 303 (1988).

${ }^{39}$ Yu. I. Latyshev, O. Laborde, P. Monceau, and S. Klaumunzer, Phys. Rev. Lett. 78, 919 (1997).

${ }^{40}$ J. Yi, M. Y. Choi, K. Park, and E.-H. Lee, Phys. Rev. Lett. 78, 3523 (1997).

${ }^{41}$ I. V. Krive, P. Sandstrom, R. I. Shekhter, S. M. Girvin, and M. Jonson, Phys. Rev. B 52, 16451 (1995).

${ }^{42}$ M. V. Moskalets, Physica E 4, 17 (1999).

${ }^{43}$ M. F. Lin and D. S. Chuu, Phys. Rev. B 57, 6731 (1998).

${ }^{44}$ T. Swahn, E. N. Bogachek, Yu. M. Galperin, M. Jonson, and R. I. Shekhter, Phys. Rev. Lett. 73, 162 (1994).

${ }^{45}$ E. N. Bogachek, Yu. M. Galperin, M. Jonson, R. I. Shekhter, and T. Swahn, J. Phys.: Condens. Matter 8, 2603 (1996).

${ }^{46}$ I. O. Kulik, Physica B 218, 252 (1996).

${ }^{47}$ O. Entin-Wohlman, A. Antony, Y. Imry, Y. Levinson, and A. Schiller, Phys. Rev. Lett. 88, 166801 (2002)

${ }^{48}$ I. O. Kulik and A. S. Shumovsky, Appl. Phys. Lett. 69, 2779 (1996).

${ }^{49}$ M. V. Moskalets, Physica E 5, 124 (1999).

${ }^{50}$ E. N. Bogachek, I. V. Krive, I. O. Kulik, and A. S. Rozhavsky, Mod. Phys. Lett. B 5, 1607 (1991).

${ }^{51}$ E. N. Bogachek, I. V. Krive, I. O. Kulik, and A. S. Rozhavsky, Phys. Rev. B 42, 7614 (1990).

${ }^{52}$ E. N. Bogachek and U. Landman, Phys. Rev. B 50, 2678 (1994).

${ }^{53}$ I. V. Krive and A. S. Rozhavsky, Int. J. Mod. Phys. B 6, 1255 (1992).

${ }^{54}$ Y. Imry, in Quantum Coherence in Mesoscopic Systems, edited by B. Kramer, Plenum Press, New York (1991). 
${ }^{55} \mathrm{~S}$. Washburn, in Mesoscopic Phenomena in Solids, edited by B. L. Altshuler, P. A. Lee, and R. A. Webb, Elsevier (1991).

${ }^{56}$ S. Washburn and R. A. Webb, Adv. Phys. 35, 375 (1986).

${ }^{57}$ I. O. Kulik, Doga: Turk. J. Phys. 27, 395 (2003).

${ }^{58}$ A. Barone, T. Hakioglu, and I. O. Kulik, "Quantum computation with Aharonov-Bohm qubits," E-print cond-mat/0203038 (2002).

${ }^{59}$ I. O. Kulik, T. Hakioglu, and A. Barone, Eur. Phys. J. B 30, 219 (2002).

${ }^{60}$ I. O. Kulik, in Towards the Controllable Quantum States: Mesoscopic Superconductivity and Spintronics, edited by H. Takayanagi and J. Nitta, World Scientific (2002).

${ }^{61}$ M. A. Nielsen and I. L. Chuang, Quantum Computation and Quantum Information, Cambridge Univ. Press (2000).

${ }^{62}$ H. F. Cheung, E. K. Riedel, and Y. Gefen, Phys. Rev. Lett. 62, 587 (1989).

${ }^{63}$ I. O. Kulik and I. K. Yanson, The Josephson Effect in Superconductive Tunneling Structures, Israel Program for Scientific Translations, Jerusalem (1972).

${ }^{64}$ Barone and G. Paterno, Physics and Applications of the Josephson Effect, Wiley, New York (1982).

${ }^{65}$ A. J. Leggett, in Chance and Matter, edited by J. Souletier, J. Vannimenus, and R. Stora, Elsevier, Amsterdam (1996), p. 395.

${ }^{66}$ K. K. Likharev, Dynamics of Josephson Junctions and Circuits, Gordon and Breach, Amsterdam (1996).

${ }^{67}$ Y. Makhlin, G. Schön, and A. Schnirman, Rev. Mod. Phys. 73, 357 (2001).
${ }^{68}$ H.-F. Cheung, Y. Gefen, E. K. Riedel, and W.-H. Shih, Phys. Rev. B 37, 6050 (1988).

${ }^{69}$ S. Latil, S. Roche, and A. Rubio, Phys. Rev. B 67, 165420 (2003).

${ }^{70}$ M. I. Vischer, B. Rejaei, and G. E. W. Bauer, Europhys. Lett. 36, 613 (1996).

${ }^{71}$ B. Nathanson, O. Entin-Wohlman, and B. Mülschlegel, Phys. Rev. Lett. 80, 3416 (1998).

${ }^{72}$ G. Montambaux, Phys. Rev. Lett. 80, 3417 (1998).

${ }^{73}$ R. E. Peierls, Quantum Theory of Solids, Clarendon Press, Oxford (1995).

${ }^{74}$ H. J. Jahn and E. Teller, Proc. R. Soc. London, Ser. A 161, 220 (1937).

${ }^{75}$ C. Kittel, Introduction to Solid State Physics, Wiley, New York (1996).

${ }^{76}$ L. N. Bulaevskii, Usp. Fiz. Nauk [Sov. Phys. Usp. 115, 261 (1975)].

${ }^{77}$ W. A. Harrison, Electronic Structure and the Properties of Solids, Cambridge (1972).

${ }^{78}$ J. M. Ziman, Principles of the Theory of Solids, Cambridge (1972).

${ }^{79}$ I. O. Kulik, in Technical Proceedings of the 2003 Nanotechnology Conference and Trade Show, edited by M. Laudon and B. Romanowicz, Computational Publications, Boston (2003), Vol. 2, p. 531.

This article was published in English in the original Russian journal. Reproduced here with stylistic changes by AIP. 